\title{
Elections fédérales 2007
}

\section{Jura}

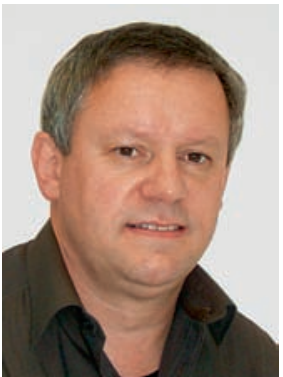

Pierre-Alain Fridez, 1957, Fontenais

Médecin spécialiste

en médecine générale FMH

Candidat au Conseil national sur la liste du Parti socialiste jurassien

En parallèle à mon engagement en faveur d'une Suisse plus sociale et plus
Le BMS offre aux médecins qui se portent candidats au Conseil national et au Conseil des Etats dans le cadre des élections fédérales du 21 octobre prochain, la possibilité de se présenter brièvement à nos lecteurs. Pour ce faire, ils sont priés d'envoyer par courriel un texte succinct accompagné des coordonnées usuelles à notre rédaction (redaktion.saez@emh.ch) d'ici au vendredi 28 septembre 2007 au plus tard afin que leur texte puisse être publié avant le week-end des élections.

\section{St. Gallen}

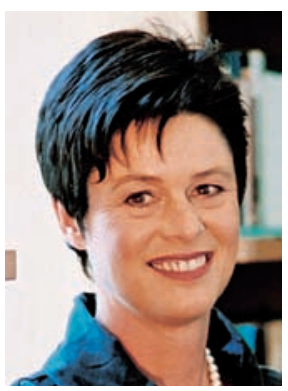

\section{Barbara Frei, 1958, St. Gallen}

Fachärztin für Allgemeinmedizin FMH

Nationalratskandidatin der FDP ouverte, de la défense des plus défavorisés et de la promotion d'une politique de développement durable respectueuse de l'environnement, je souhaite contribuer à la défense et à la revalorisation de la médecine de premier recours dans notre système de soins. Notre rôle central et primordial doit être reconnu dans une perspective de qualité et d'universalité des soins, d'économies et de proximité. Cette reconnaissance permettra l'émergence de vocations et d'éviter la disparition programmée du plus beau métier du monde: médecin de famille.

\section{St. Gallen}

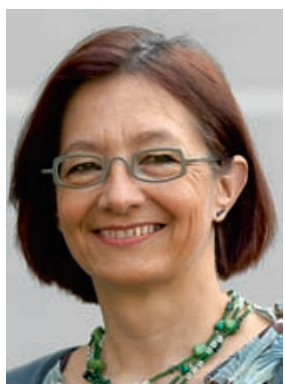

Yvonne Gilli, 1957, St. Gallen

Fachärztin für Allgemeinmedizin FMH, Homöopathie SVHA, TCM ASA

Kandidatin für den Ständerat und den Nationalrat der Grünen

Als Mitglied einer Kerngruppe der Ärzte und Ärztinnen für Umweltschutz beschäftige ich mich intensiv mit Erkran-
Unser Gesundheitswesen soll bestmögliche Leistungen bieten und gleichzeitig für alle bezahlbar bleiben. Die Bevölkerung muss mehr Eigenverantwortung für die Gesundheit übernehmen. Ein Ausbau des geltenden Systems der freiwählbaren Franchisen mit entsprechenden Prämienreduktionen stärkt die Selbstverantwortung. Die Ausweitung des Leistungskatalogs in der Grundversicherung soll vermieden werden. Im Spitalbereich ist das Schaffen wirtschaftlicher Rahmenbedingungen besonders wichtig.

Die Hausarztmedizin als wichtiger Grundpfeiler der ärztlichen Versorgung soll gestärkt werden; dazu muss der Aus- und Weiterbildung in Hausarztmedizin besondere Beachtung geschenkt werden.

kungen als Folge von Umweltverschmutzung und Anwendung neuer Technologien, deren gesundheitliche Auswirkungen ungenügend erforscht sind. Mit einer wirksamen Umweltpolitik wird auch die Prävention von umweltbedingten Erkrankungen in Bern zu einem zentralen Thema werden. Daneben liegt mir die ärztliche Standespolitik am Herzen. Auch in Zukunft kann die freie Praxistätigkeit sowohl in der Grundversorgung als auch in der Spezialmedizin erfolgreich sein. Ich engagiere mich für Ärztenetzwerke, die offen sind für zukunftsgerichtete Veränderungen. Dabei ist es mir wichtig, der ärztlichen Ethik und Unabhängigkeit verpflichtet zu bleiben. 
Vaud

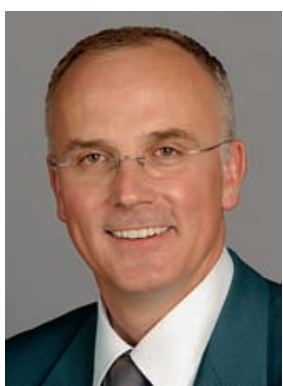

Charles Favre, 1957, Echallens

Médecin généraliste, Conseiller national

Candidat au Conseil national

et au Conseil des Etats du Parti radical

Les débats de ces dernières années sur la politique de la santé ont montrés combien il est indispensable que tous les partenaires soient de même force là où se prennent les décisions majeures, à savoir au Parlement fédéral. Les prestataires de santé, médecins et hôpitaux notamment, y sont aujourd'hui sous-représentés. Il faut que cela change!

Pour cela il est nécessaire que les prestataires soient représentés par des personnes bénéficiant d'une large expérience à la fois médicale et politique. Il y va du développement à long terme d'une médecine de qualité, respectueuse de l'être humain, et au service de tous.

\section{Vaud}

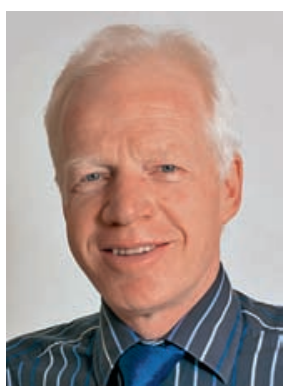

Jacques-André Haury, 1952, Lausanne

Médecin spécialiste en ORL

et chirurgie cervico-faciale FMH

Candidat au Conseil national du Mouvement Ecologie libérale

Au cœur du système de santé: la relation entre le patient et son médecin. Dans tout l'édifice des intervenants en matière de soins, seul le médecin accepte de prendre sur lui la responsabilité de son patient.

Certains voudraient que le médecin travaille sur mandat d'un «trieur», par exemple un infirmier ou une infirmière; ou que l'assureur puisse intervenir pour diriger le patient; ou que les pouvoirs publics établissent des directives précises en matière de dispensation ou de rationnement des soins. Toutes ces démarches transforment une profession libérale en une simple relation producteur-consommateur. Ayant combattu la «caisse unique», nous combattrons également cette dérive «économiste» pratiquée notamment par Santésuisse.

\section{Vaud}

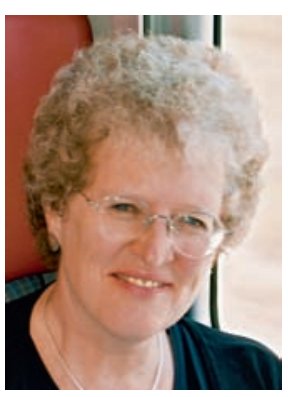

Marianne Huguenin, 1950, Renens

Médecin spécialiste de médecine générale $\mathrm{FMH}$, Conseillère nationale

Candidate au Conseil national du Parti Ouvrier et Populaire (A Gauche toute!)

Dans ma formation et ma pratique, j'ai privilégié une médecine globale. J'ai été 17 ans installée dans la ville dont je suis maintenant la syndique, Renens.
Elue en 2003 à Berne, j'ai renoncé à ma pratique. L'introduction du TARMED, l'emprise grandissante des caisses-maladie et du travail administratif m'ont aidé à faire ce choix.

La défense d'une médecine de qualité et de l'indépendance des médecins va de pair pour moi avec la défense des assurés et des malades. Je me suis inquiétée à Berne de la pénurie qui menace la médecine de premier recours. J'ai toujours été contre la clause de besoin, contre la suppression de l'obligation de contracter. Je suis opposée au libre choix des hôpitaux, comme d'ailleurs au financement par cas qui va les entraîner dans la logique de la concurrence. www.mariannehuguenin.ch.

\section{Vaud}

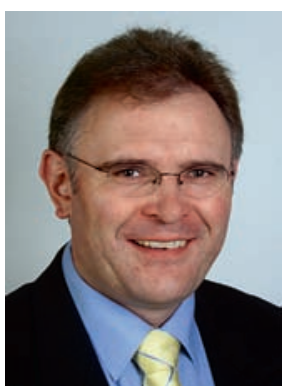

\section{Roland Oppliger, 1968, Nyon}

Médecin spécialiste en médecine interne $\mathrm{FMH}$ et en allergologie et immunologie clinique $\mathrm{FMH}$

Candidat au Conseil national du Parti évangélique (PEV)
Les orientations politiques multiples et les divergences d'intérêt des médecins sont des facteurs qui affaiblissent la position de notre corps de métier malgré qu'il soit bien représenté à Berne. Il est donc impératif de créer un réel «lobby» des médecins pour être entendu et faire face à celui d'autres milieux impliqués dans le système de santé. Le maintien de l'obligation de contracter, du libre choix du médecin et d'une médecine de premier recours de qualité continueront à être prioritaires tout comme la formation de notre relève. 